\title{
Bone fragility and matrix hypermineralization are rescued in homozygous OI BrtI mice mutants
}

\author{
Nadja Fratzl-Zelman ${ }^{*}{ }^{*}$, Kenneth M. Kozloff ${ }^{2}$, Jeff Meganck², Adi Reich ${ }^{3}$, \\ Paul Roschger ${ }^{1}$, Wayne Cabral ${ }^{3}$, Klaus Klaushofer ${ }^{1}$, Joan Marini ${ }^{3}$ \\ ${ }^{1}$ Ludwig Boltzmann Institute of Osteology at Hanusch Hospital of WGKK and AUVA Trauma Centre Meidling, \\ 1st Med. Dept. Hanusch Hospital, Vienna, Austria \\ ${ }^{2}$ Orthopaedic Research Laboratories, Department of Orthopaedic Surgery, University of Michigan, Ann Arbor, MI, USA \\ ${ }^{3}$ Bone and Extracellular Matrix Branch, NICHD, NIH, Bethesda MD, USA
}

\section{Introduction:}

Classical Osteogenesis imperfecta (OI) is caused by mutations in one of the the two genes encoding for type I collagen.

OI is associated with low bone mass and abnormally high bone matrix mineralization.

The Brtl/+ OI mouse is a knock-in mouse model for non-lethal OI type IV caused by a glycine substitution in one COL1A1 allele.

Heterozygous Brtl/ + mutants have:

- the glycine substitution in one COL1A1 allele

- $30 \%$ perinatal lethality

- small size, decreased BMD and increased bone fragility

$\rightarrow$ OI phenotype

Ref: Forlino, A et al., J Biol Chem 1999 Kozloff, KM et al., J Bone Miner Res 2004

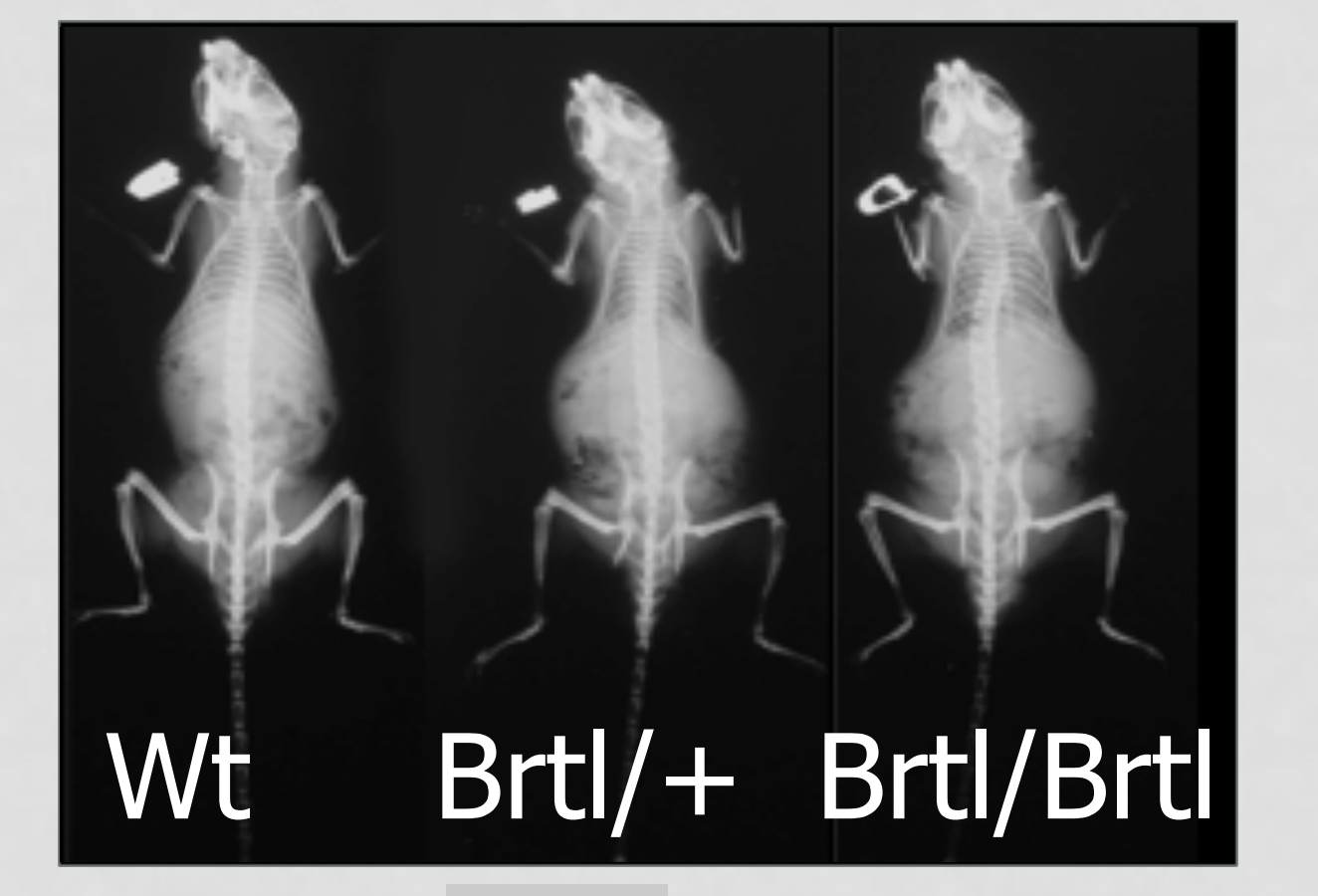

$\mathrm{Brtl} /+\quad \times \quad \mathrm{Brtl} /+$
Homozygous Brtl/Brtl mutants have:

- the glycine substitution in both COL1A1 alleles

- normal survival rates

- a rescued phenotype with normal bone fragility. $\rightarrow$ minimal OI phenotype

Brtl/+

Brtl/Brtl

Is the rescued bone fragility in Brtl/Brtl mutants reflected by normalized bone matrix mineralization?

Method: quantitative Backscattered Electron Imaging (qBEI) to evaluate Bone Matrix Density distribution (BMDD) in femoral bone in 2month-old mutants at the metaphysis, epiphysis \& corticalis. $\mathrm{CaMean}=$ mean calcium concentration of the bone matrix CaPeak=most frequent calcium concentration of the bone matrix Ref: Roschger, P et al., Bone 2008 BMDD in metaphyseal cancellous bone heterozygous Brtl/+ mutant homozygous Brtl/Brtl mutant

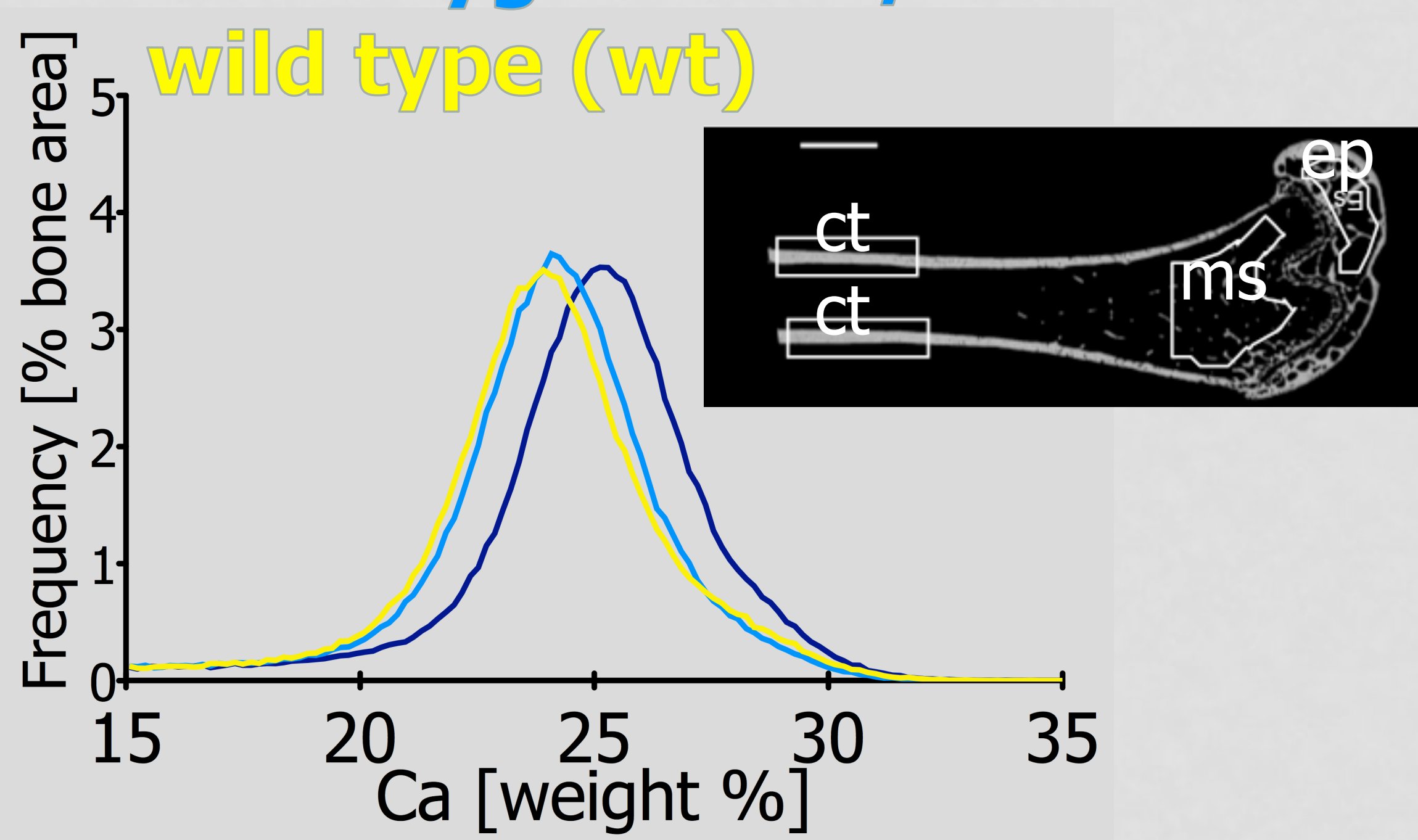

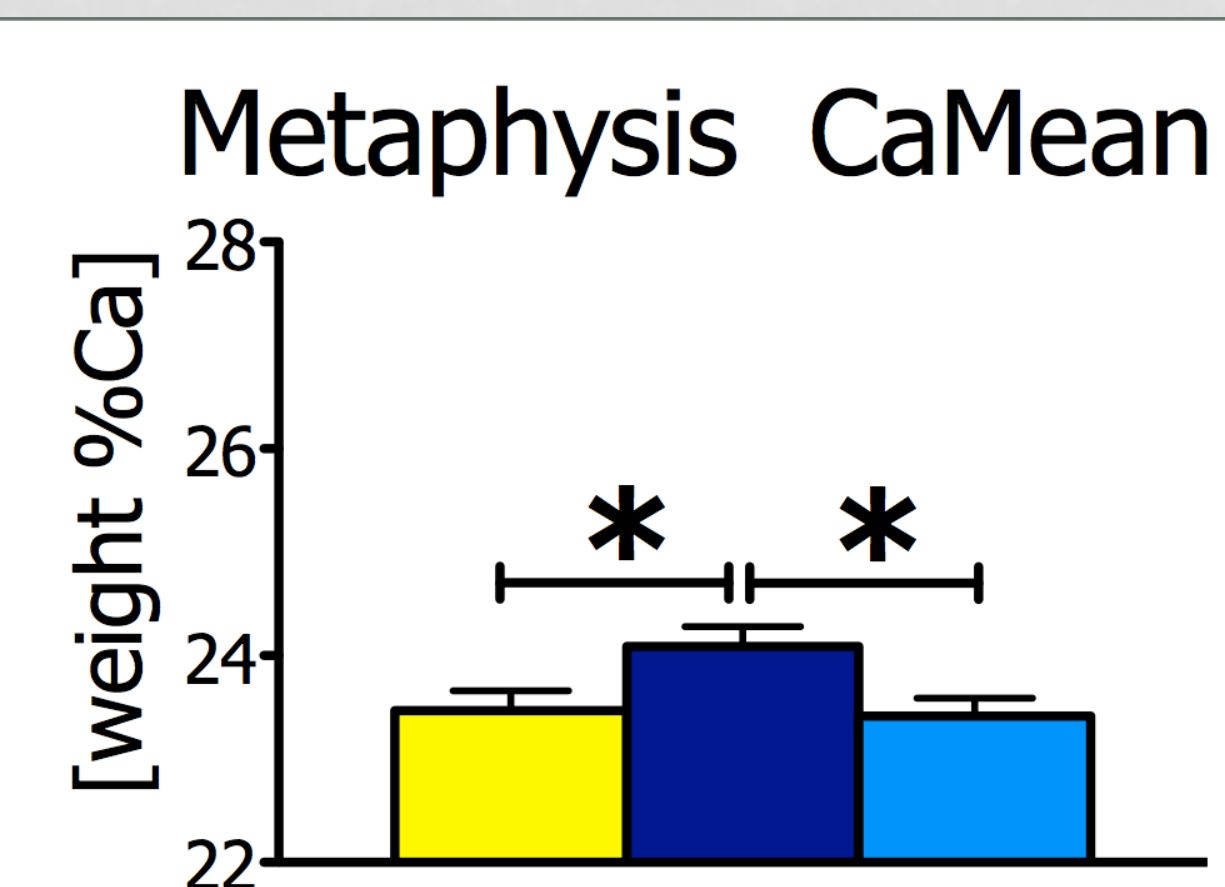

Metaphysis CaPeak

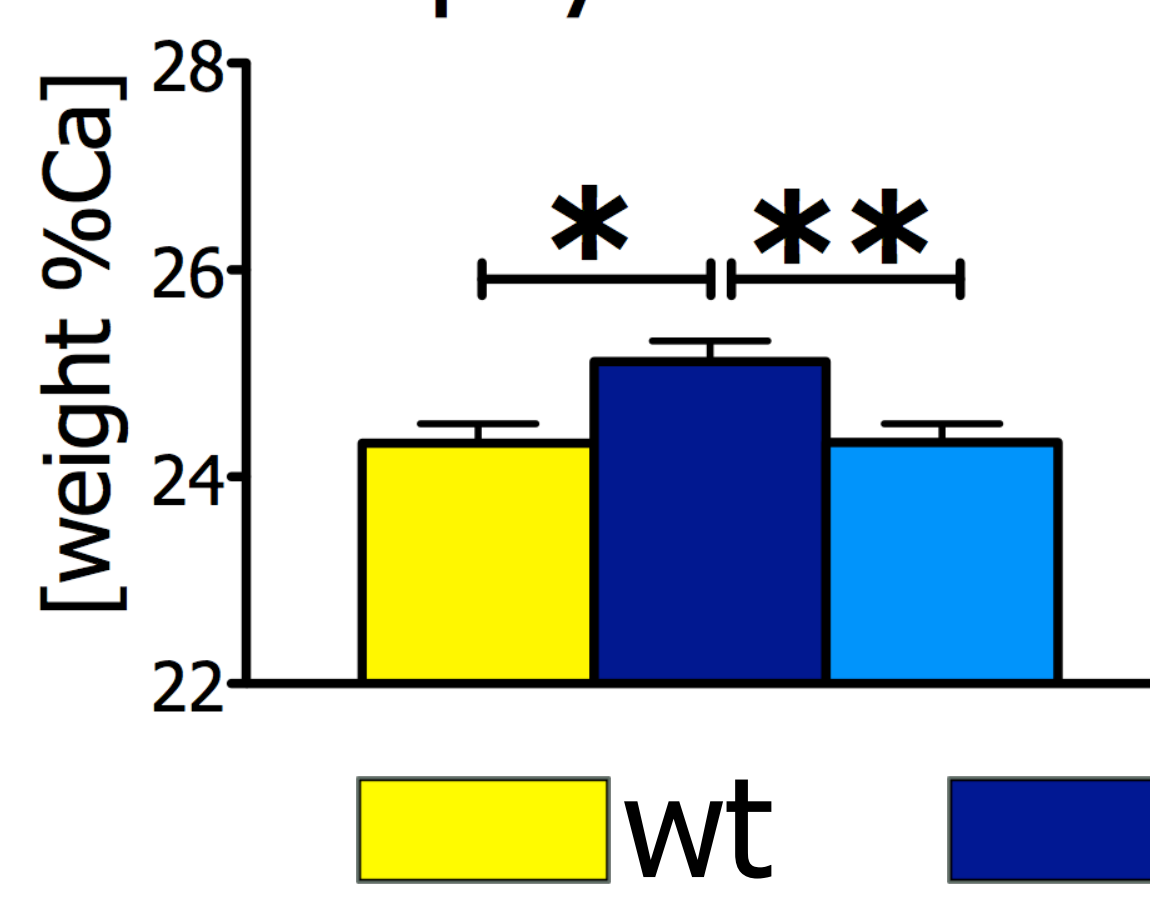

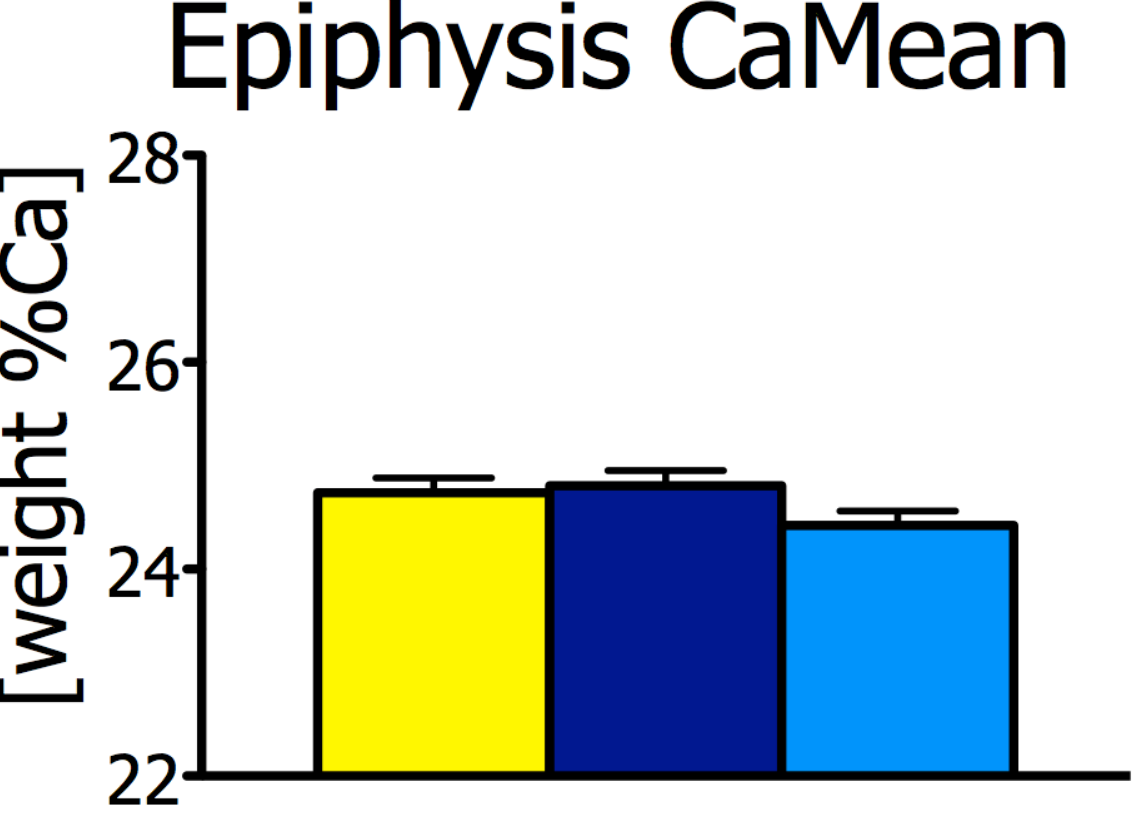

Epiphysis CaPeak

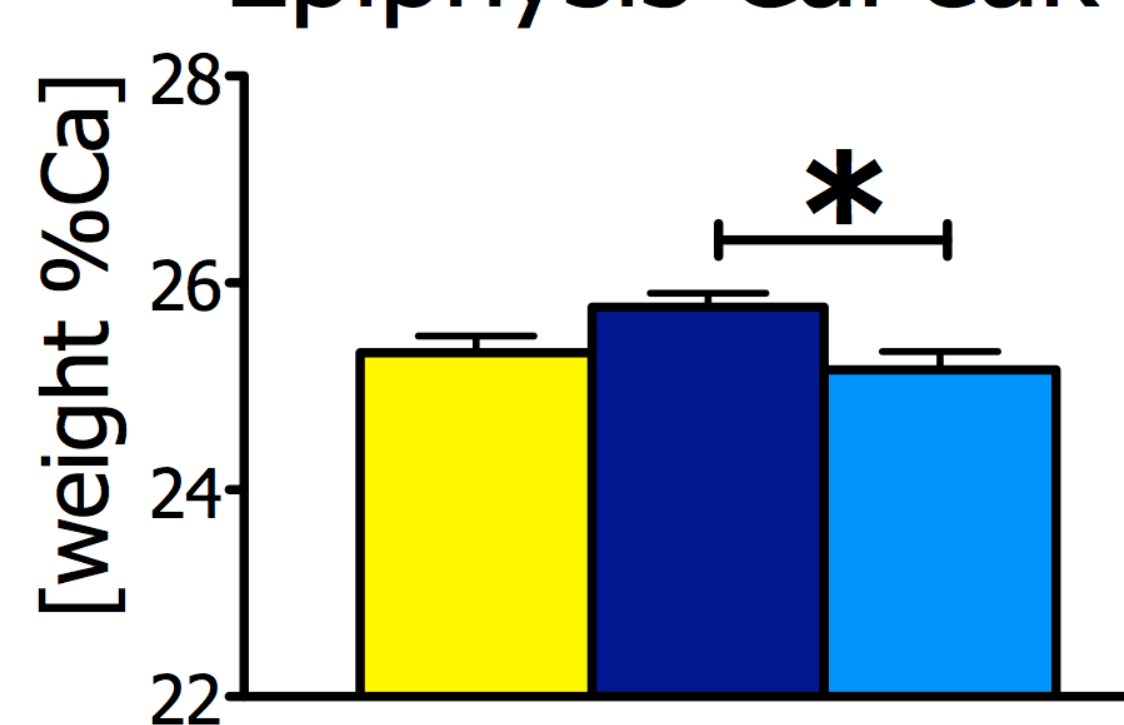

brtl/+ $\square$ brtl/brtl

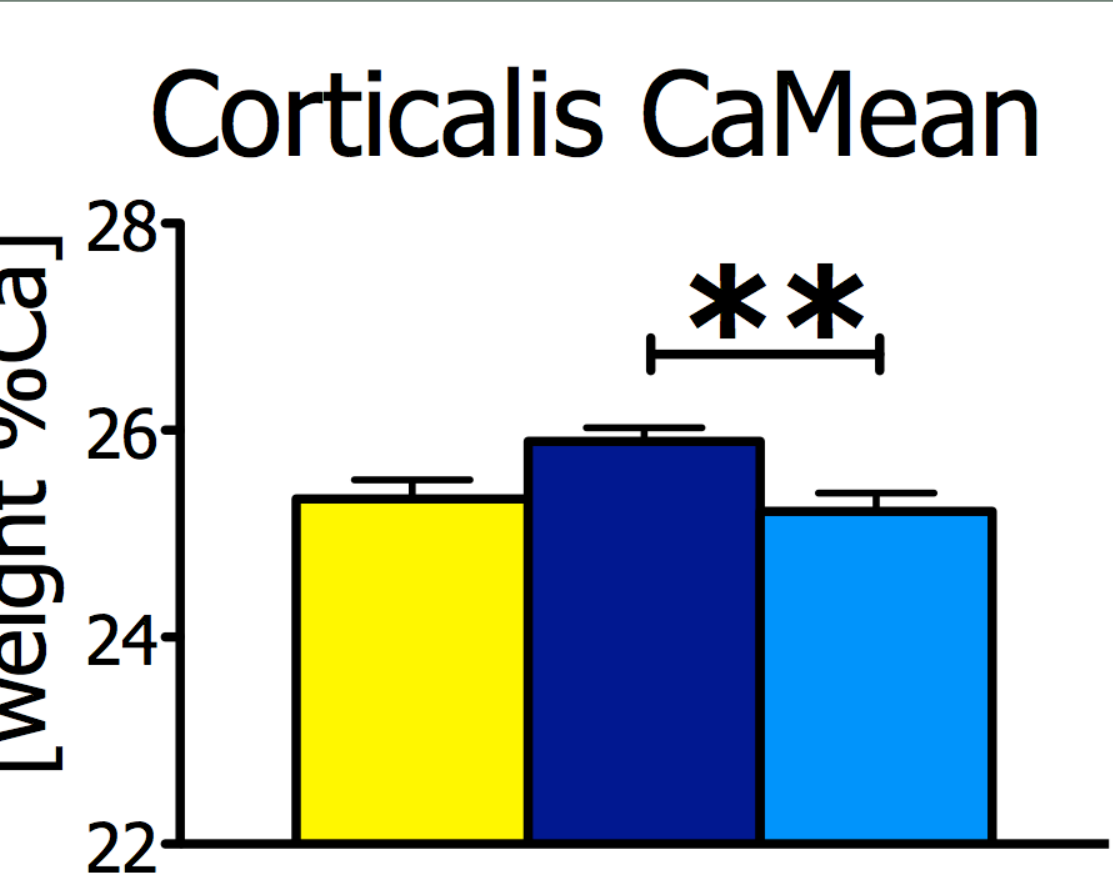

Corticalis CaPeak

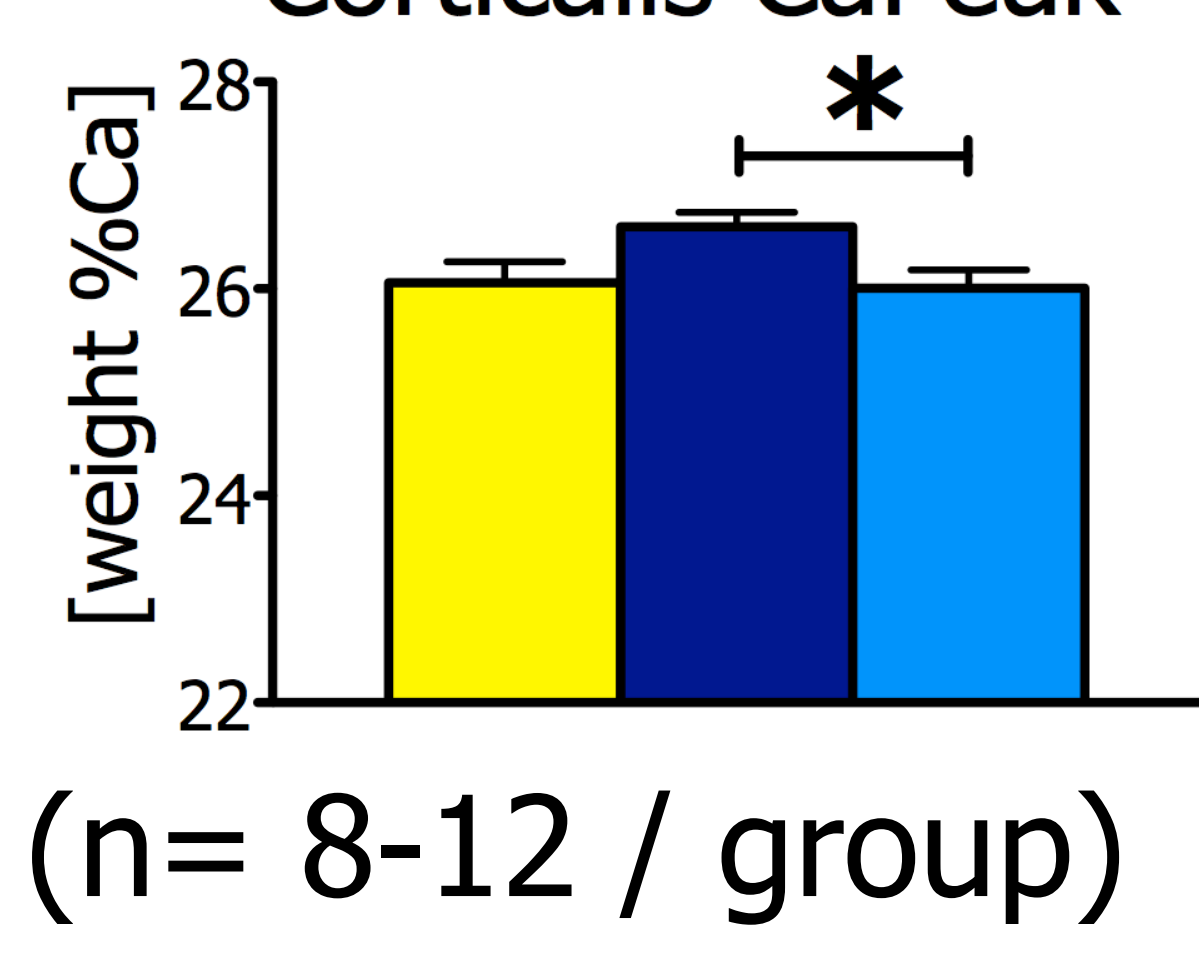

Are bone fragility and BMDD in Brtl/Brtl mutants rescued because of matrix homogeneity?

Heterozygous Mov13/+ mutants have

$\rightarrow$ a null COL1A1 allele (haploinsufficency)

$\rightarrow 2$ normal $\alpha 1$ (I) chains,

$\rightarrow 50 \%$ matrix insufficiency

$\rightarrow$ a moderate OI phenotype
Homozygous Brtl/Mov mutants have

$\rightarrow 2$ mutant $\alpha 1$ (I) chains

$\rightarrow 67 \%$ matrix insufficiency

$\rightarrow$ Phenotype ? $\mu \mathrm{CT}$ :

Cross Sectional Area

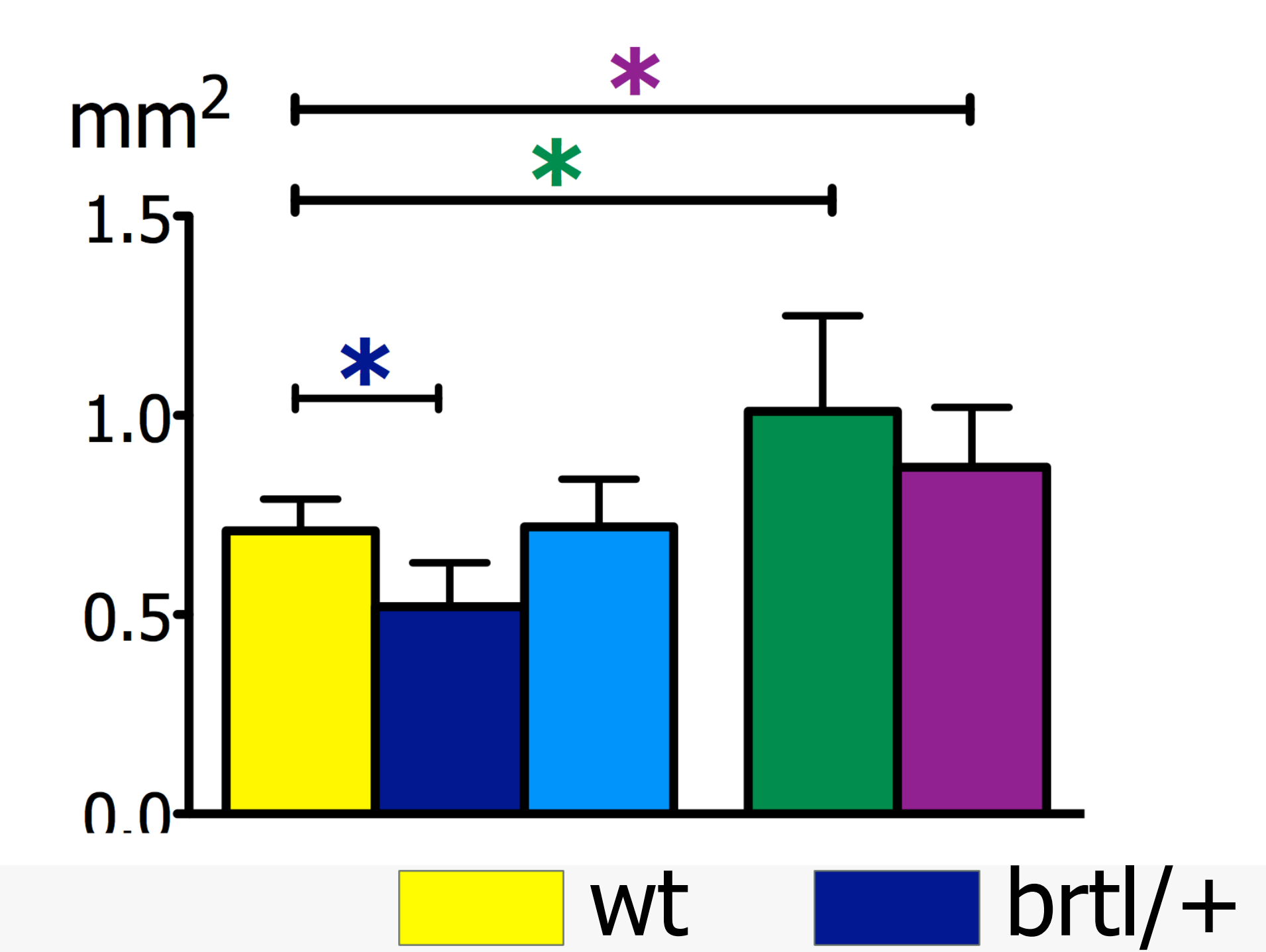

4-point bending:

Ultimate Load

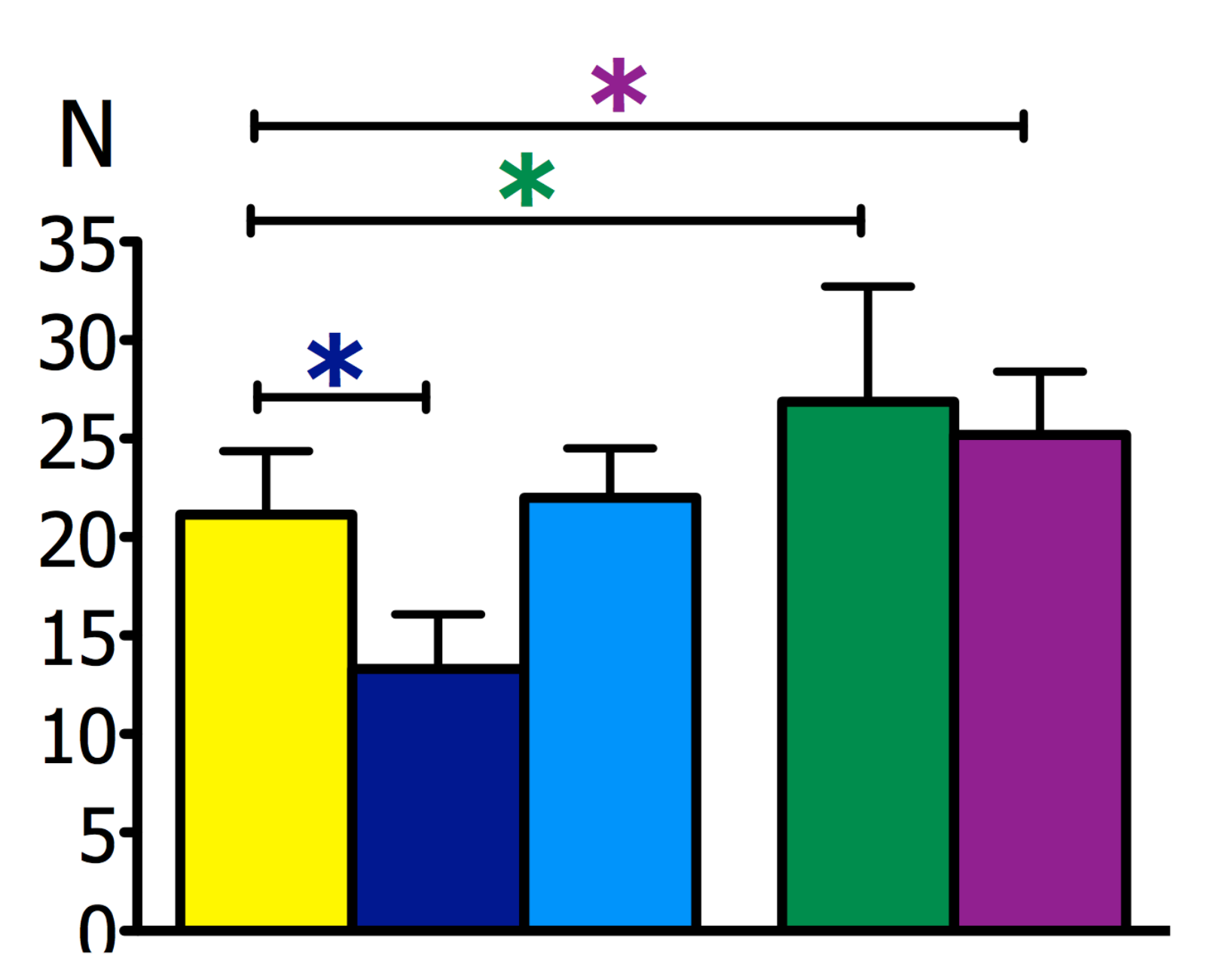

$\mathrm{brtl} / \mathrm{brtl}$
Mov13/+

Brtl/+

\section{Brtl/Mov13}

qBEI:

CaMean Corticalis

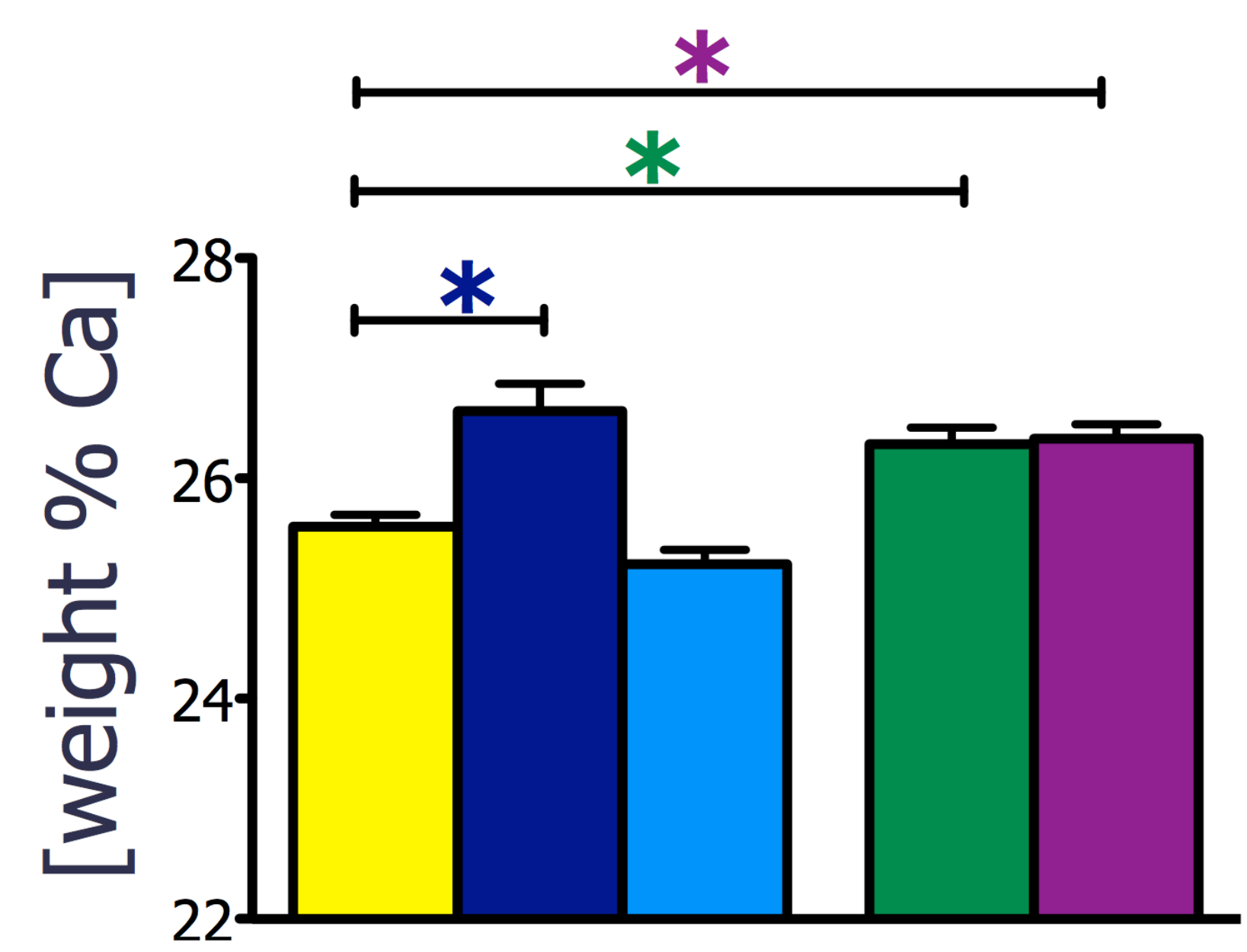

brtl/Mov13 ( $n=8-12$ / group)

Cross-sectional area and ultimate load are lower in Brtl/ + , similar in Brtl/Brtl and significantly higher in Mov/+ and and Brtl/Mov13. This indicates that the increased load to fracture in Mov13/+ and $\mathrm{Brtl} /$ Mov13 is due to altered bone geometry.

CaMean and CaPeak are similar in Brtl/Brtl and WT but significantly higher in all other groups compared.

This indicates an OI phenotype of bone material in $\mathrm{Brtl} /+$ Mov13/+ and Brtl/Mov13 but a minimal one in Brtl/Brtl.

\section{Conclusion:}

These results indicate that in Brtl/Brtl mice both mechanical properties and hypermineralization of the matrix are rescued by homozygosity, which may be caused by homogeneity of matrix with mutant collagen, while Brtl/Mov13 mutants have increased ultimate load due to increased cross-sectional area compared to WT. However, the hypermineralization associated with severe matrix insufficiency is not normalized despite the bone size adaptation. 